\title{
Single and bilayer bismuthene: Stability at high temperature and mechanical and electronic properties
}

\author{
E. Aktürk,,${ }^{1,2,3, *}$ O. Üzengi Aktürk, ${ }^{2,3,4}$ and S. Ciraci ${ }^{3, \dagger}$ \\ ${ }^{1}$ Department of Physics, Adnan Menderes University, 09100, Aydin, Turkey \\ ${ }^{2}$ Nanotechnology Application and Research Center, Adnan Menderes University, Aydin 09010, Turkey \\ ${ }^{3}$ Department of Physics, Bilkent University, 06800 Ankara, Turkey \\ ${ }^{4}$ Department of Electric and Electronic Engineering, Adnan Menderes University, 09100, Aydın, Turkey
}

(Received 18 March 2016; revised manuscript received 16 May 2016; published 20 July 2016)

\begin{abstract}
Based on first-principles phonon and finite temperature molecular dynamics calculations including spinorbit coupling, we showed that free-standing single-layer phases of bismuth, namely buckled honeycomb and asymmetric washboard structures named as bismuthene, are stable at high temperature. We studied the atomic structure, mechanical, and electronic properties of these single-layer bismuthene phases and their bilayers. The spin-orbit coupling is found to be crucial in determining lattice constants, phonon frequencies, band gaps, and cohesion. In particular, phonons of 3D hexagonal crystal, as well as those of single-layer bismuthene phases, are softened with spin orbit coupling. By going from 3D hexagonal crystal to free-standing single-layer structures, $2 \mathrm{D}$ hexagonal lattice is compressed and semimetal is transformed to semiconductor as a result of confinement effect. On the contrary, by going from single-layer to bilayer bismuthenes, the lattice is slightly expanded and fundamental band gaps are narrowed. Our results reveals that interlayer coupling in multilayer and 3D Bi crystal is crucial for topologically trivial to nontrivial and semimetal to semiconductor transitions.
\end{abstract}

DOI: 10.1103/PhysRevB.94.014115

\section{INTRODUCTION}

Recent theoretical and experimental studies have revealed a new class of single-layer (SL) structures or very thin films of group $\mathrm{V}$ elements with a wide range of physical properties. These are nitrogene [1], phosphorene [2,3], arsenene [4], and antimonene [5,6]. Depending on the row numbers of their constituent atoms they can form buckled honeycomb $[1,3-5]$ as well as either symmetric [3,4] or asymmetric washboard [5] structures. They remain stable above the room temperatures [1,5]. Because of 10 valence electrons per primitive cell, the counterparts of well-known $\pi$ and $\pi^{*}$ bands of planar honeycomb structure of graphene [7] and of buckled honeycomb structure of silicene and germanene [8,9] leading to Dirac cones are filled and separated from the conduction band by a gap; the width of it depends on the row number.

Bismuth, the last and heaviest element of group V column or pnictogens, forms a three-dimensional (3D) hexagonal crystal. This quasilayered crystal and its alloys exhibit a number of peculiarities and mysterious features, which have attracted the interest of many researchers for many years [10-13]. Recently, the semimetal-semiconductor and trivial-to-topological transitions [14-17] under strain have been subjects of active studies [18]. Additionally, the binary compounds of $\mathrm{Bi}, \mathrm{Bi}_{2} X_{3}$, and binary alloys $\mathrm{Bi}_{x} X_{1-x}$, where $X=\mathrm{Se}, \mathrm{Sb}$, and $\mathrm{Te}$, are found to be 3D topological insulators $[19,20]$. Small band gap and large spin-orbit coupling in Bi crystals and Bi compounds are primary features which can make these crystals topologically important.

Even before single-layer structures of N, P, As, and $\mathrm{Sb}$ have been brought into the focus, thin films of $\mathrm{Bi}$ crystal

\footnotetext{
*ethem.akturk@adu.edu.tr

†ciraci@fen.bilkent.edu.tr
}

grown on selected substrates have been actively investigated to explore their two-dimensional (2D) topological insulator behavior together with surface and edge states [21-28]. As early as 1997, ultra thin film generated from the Bi(111) surface based on the so-called distorted A7 phase was deduced [29]. This phase reflects the honeycomb structure from the top view, while $\mathrm{Bi}$ atoms at alternating corners of hexagon are buckled. Later, based on scanning tunneling microscopy and electron diffraction experiments a $2 \mathrm{D}$ phase of $\mathrm{Bi}$, pseudocubic 012-oriented allotrope (so-called A17 phase), was revealed in thin Bi films grown on the $\operatorname{Si}(111)-7 \times 7$ surface, which were stable up to critical thickness of four atomic layers at room temperature [21]. On the basis of first-principles calculations, this phase of $\mathrm{Bi}$, named as the washboard structure now, is identified as a "black-phosphorus-like" ultra thin film [21]. Intense theoretical efforts have been devoted to interpret angleresolved photoemission and scanning-tunneling spectroscopy data obtained from the thin films of diverse thicknesses constructed from these two phases.

Electronic structure of A7- and A17-like thin films have been studied with spin-orbit coupling (SOC) [22,23] leading to a large variation of electronic properties ranging from narrow band gap semiconducting to semimetallic and metallic states. Rashba-type spin splitting of the surface states due to strong SOC in Bi and monitoring of surface states as a function of film thickness were other important findings [22]. It has been proposed that the stable nontrivial $Z_{2}$ topology is retained by the concurrent band gap inversions at multiple time reversal invariant $\mathbf{k}$ points of hexagonal $\mathrm{BZ}$ with the increasing thickness of the films [25]. Namely, all these ultrathin A7 films were found to be topological insulator [25]. The effects of strain and electric field on the electronic structure of a single-layer A7 structure are investigated to show that the structure is a robust 2D topological insulator against strain and electrical field [27]. In addition, the electronic properties 
of $X \mathrm{Bi}$ binary compound $(X=\mathrm{B}, \mathrm{Al}, \mathrm{Ga}$, and $\mathrm{In})$ and hydrogenated $\mathrm{Bi}(111)$ films [30] have been explored within the perspective of topological insulator.

As the quest for 2D topological insulator phases focused on the electronic structure of $\mathrm{Bi}$ ultrathin films, ultrathin films of diverse atomic structures have been unveiled with diverse electronic properties varying with film thickness. In the meantime, stabilities of those films, their mechanical (elastic) properties and energetics have not been considered yet. This study presents an extensive analysis of the stability of free-standing monolayer structures of $\mathrm{Bi}$. These are buckled honeycomb $(b-\mathrm{Bi})$, symmetric washboard $(w$-Bi $)$, and asymmetric washboard ( $a w-\mathrm{Bi})$ structures and are identified as bismuthene like the aforementioned monolayer structures of other group $\mathrm{V}$ elements. The first one is similar to the structure which was specified as A7 Bi(111) "bilayer" due to the two atomic planes occurring after buckling. The second one is reminiscent of the A17, 2D black-phosphorous-like structure [21]. In our earlier study, similar structures as $b-\mathrm{Sb}$, $w$-Sb, and $a w$-Sb were treated for 2D SL antimony, named as antimonene.

In this paper, we first consider the optimized atomic structure of 3D hexagonal $\mathrm{Bi}$ and the corresponding electronic structure which is corrected with hybrid functional HSE method [31] including SOC. This constitutes a reference point for our further study on SL bismuthene phases. Then we determine the equilibrium structure of these free-standing SL $b$ - $\mathrm{Bi}, w-\mathrm{Bi}$, and $a w$ - $\mathrm{Bi}$ phases at $T=0 \mathrm{~K}$ by minimization of total energy and atomic forces. We examine their stability against small displacements of $\mathrm{Bi}$ atoms from their equilibrium sites at $T=0 \mathrm{~K}$ based on $a b$ initio calculations of vibrational frequencies and phonon dispersion curves with and without SOC. Here we deduce a crucial effect of SOC. High temperature stability of these three structures is examined using finite temperature ab initio molecular dynamic (MD) calculations. The mechanical properties of these three phases are investigated in terms of in-plane stiffness and Poisson's ratio, which are crucial for the robustness of these structures and variation of physical properties under strain. Then, we calculate the electronic energy structure of these SL phases by including SOC and by correcting with HSE [31] and compare them with previous calculations. Finally, we investigate ultrathin films consisting of two SL $b-\mathrm{Bi}, a w-\mathrm{Bi}$ structures.

\section{METHOD}

Our theoretical analysis and predictions are obtained from first-principles pseudopotential calculations based on the spin-polarized density functional theory (DFT) [32] within the generalized gradient approximation including spin-orbit coupling (SOC). Here we used projector-augmented wave (PAW) potentials [33] and the exchange-correlation potential is approximated with the Perdew-Burke-Ernzerhof (PBE) functional [34]. The electronic band structures, vibrational frequencies, and phonon dispersion relations of bulk $\mathrm{Bi}$ and single-layer Bi phases have been investigated with and without SOC. Numerical calculations were carried out using the VASP software [35]. A plane-wave basis set with kinetic energy cutoff is taken to be $\hbar^{2}|\mathbf{k}+\mathbf{G}|^{2} / 2 m=250 \mathrm{eV}$. The
Brillouin zone (BZ) was sampled in the $\mathbf{k}$ space within the Monkhorst-Pack scheme [36] using $(15 \times 15 \times 1)$ mesh. Atomic positions and lattice constants were optimized using the conjugate gradient method, where the total energy and atomic forces were minimized. The energy convergence value between two consecutive steps was chosen as $10^{-5} \mathrm{eV}$, and a maximum force of $0.002 \mathrm{eV} / \AA$ was allowed on each atom. Pressures on the lattice unit cell are decreased to values less than $0.1 \mathrm{kB}$. Gaussian type Fermi-level smearing method is used with a smearing width of $0.01 \mathrm{eV}$.

The cohesive energies of 2D SL bismuthene phases are calculated using the expression, $E_{C}=E_{T}$ [Bi atom $]-$ $E_{T}$ [SL phase] in terms of the spin-polarized total energies of a single $\mathrm{Bi}$ atom and the structure optimized total energy of SL phases. Here $E_{C}>0$ indicates that the formation of the SL phase is favorable energetically. The energetics of the SL phase relative to the 3D crystal of $\mathrm{Bi}$ with hexagonal lattice are deduced by the calculation of the formation energy at $T=0 \mathrm{~K}, E_{f}=E_{C}[\mathrm{SL}$ phase $]-E_{C}[3 \mathrm{D} \mathrm{Bi}]$. Here $E_{f}<0$ indicates that the SL phase corresponds to a local minimum on the Born-Oppenheimer (BO) surface.

The energy bands of 3D Bi crystal, SL $b-\mathrm{Bi}, w-\mathrm{Bi}$, and $a w$-Bi structures are calculated by using PBE including SOC. Since the band gaps are underestimated by standard DFT, we also carried out calculations to correct the PBE values using the screened hybrid functional method [31] in HSE06 level including SOC. HSE corrections typically open the PBE band gap. In this study, HSE functional is used with the default mixing parameter $\alpha=0.25$. Additionally, the effect of $\alpha$ on the fundamental band gap is investigated for $\alpha=0,0.50,0.75$, and 1.0. The frequencies of crystal vibrations and phonon dispersion curves are obtained by diagonalizing the dynamical matrix [37]. The atomic forces in the dynamical matrices are calculated by displacing certain atoms in the $5 \times 5 \times 5$ supercell for $3 \mathrm{D}$ Bi crystal and $5 \times 5 \times 1$ supercells for SL structures. High temperature $a b$ initio molecular dynamics (MD) calculations are performed using the Verlet algorithm where Newton's equations are integrated with time steps of 2 fs. We used a Nosè thermostat [38].

\section{3D BISMUTH CRYSTAL}

Bismuth having 3D crystal with hexagonal lattice has rhombohedral structure and $R \overline{3} m$ space group. This crystal displays a quasilayered formation like antimony [5] and can provide evidences that $\mathrm{Bi}$ can form stable ultrathin even SL structures. As a matter of fact, ultra thin films of Bi have been grown on selected substrates [21,23,29]. In Fig. 1(a) crystal structure, a unit cell together with lattice constants and other relevant structural parameters are shown. The lattice constants optimized by using PBE without SOC are $a=b=$ $4.59 \AA, c=12.03 \AA$, Bi-Bi bond $d_{1}=3.11 \AA$, and equilibrium buckling $\Delta=1.63 \AA$. These values change to $a=b=4.64 \AA$, $c=12.17 \AA, d_{1}=3.13 \AA, \Delta=1.61 \AA$, and the smallest interlayer distance $\Delta z=2.44 \AA$ upon optimization with PBE+SOC. Hence, significant effects of SOC are deduced not only on electronic energy bands but also on crystal structure. Nonetheless, PBE+SOC optimized lattice constants are $2-3 \%$ larger than the experimental values. Earlier, local density approximation (LDA) including SOC predicted the 

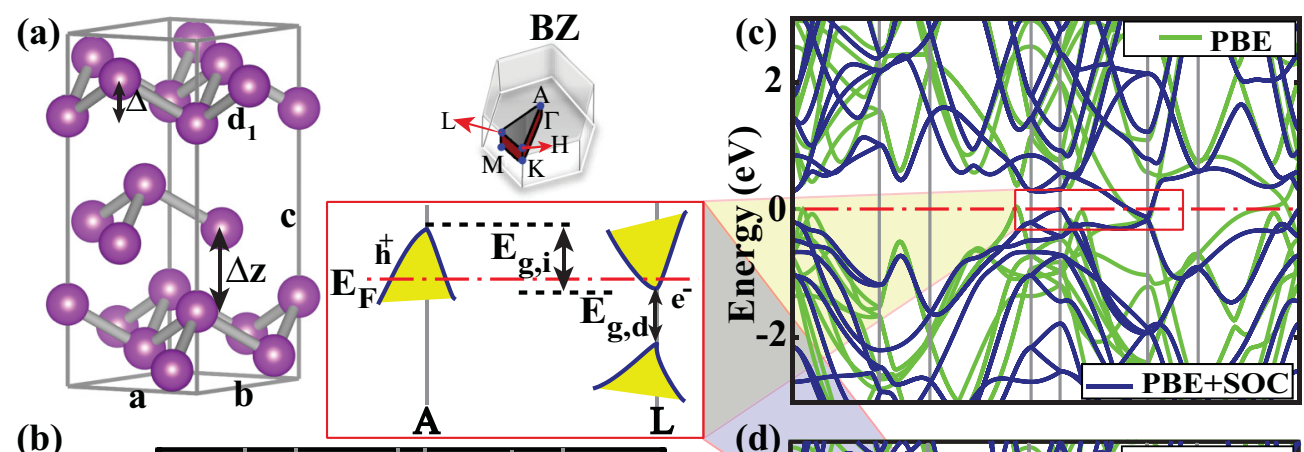

(b)
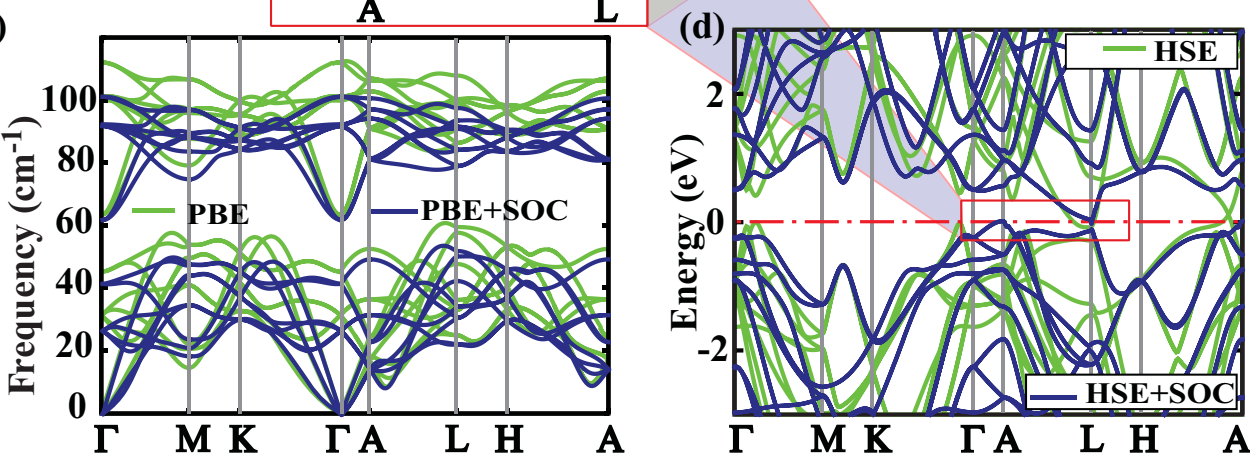

FIG. 1. 3D Bi crystal: (a) Hexagonal unit cell of 3D Bi crystal with lattice constants $a=b, c$ and relevant structure parameters $\Delta$ and $\Delta z$. (b) Phonon dispersion curves calculated with SOC (blue lines) and without SOC (green lines) show the effect of spin-orbit coupling on crystal vibrations. (c) Electronic energy band structure of 3D Bi crystal in equilibrium calculated by PBE with SOC (blue lines) and without SOC (green lines) are presented along major symmetry directions of the hexagonal Brillouin zone. The Brillouin zone and highlighted bands in the rectangle with direct $E_{g, d}$ and inverted indirect $E_{g, i}$ band gaps are shown by inset. (d) Electronic energy band structure calculated by HSE with SOC (blue lines) and without SOC (green lines). The atomic structure and lattice constants are optimized by PBE+SOC.

lattice constants as $a=b=4.58 \AA, c=12.17 \AA$ [39]. The experimental values $[21,40]$ are $a=b=4.54 \AA, c=11.81 \AA$, and equilibrium buckling $\Delta=1.59 \AA$. Here, the cohesive energy is calculated as $E_{C}=1.37 \mathrm{eV}$ per atom, which increased to $2.09 \mathrm{eV}$ /atom after SOC included. Notably, the increase of the cohesive energy as a result of SOC is significant. Its experimental value [41] is given as $E_{C}=2.18 \mathrm{eV} /$ atom.

In Fig. 1(b) the phonon frequencies and dispersion curves $\Omega(\mathbf{k})$ calculated along major directions of $\mathrm{BZ}$ are shown. We note that the frequencies of optical and acoustical modes are softened upon the inclusion of spin-orbit coupling in interatomic forces. Experiments [42] on a Bi(111) slab on the $\mathrm{Si}(111)$ surface measured frequencies of $E_{g}$ and $A_{1 g}$ modes as $\Omega=74 \mathrm{~cm}^{-1}$ and $\Omega=102 \mathrm{~cm}^{-1}$, respectively, which are close to the values of $\Omega=62 \mathrm{~cm}^{-1}$ and $\Omega=102 \mathrm{~cm}^{-1}$ calculated in this paper. These calculated frequencies also agree with earlier calculations $[43,44]$ predicting the frequency of $E_{g}$ in the range of $66 \mathrm{~cm}^{-1}<\Omega<69 \mathrm{~cm}^{-1}$ and of $A_{1 g}$ in the range of $99 \mathrm{~cm}^{-1}<\Omega<103 \mathrm{~cm}^{-1}$.

The electronic energy structure of 3D Bi crystal has been an active subject of study because of its topological character. Whether this crystal is topologically trivial $[10,40,45]$ or nontrivial [13] has been attributed to the conflicts between band gaps predicted by DFT calculations and very small band gaps (11 to $15.3 \mathrm{meV}$ for direct band gap $E_{g, d}$ and -32 to $-38.5 \mathrm{meV}$ for inverted indirect band gap, $E_{g, i}$ ) measured experimentally [46]. This controversy brought the electronic structure calculations of Bi crystal into focus. Using experimentally determined lattice constants and including the SOC throughout the self-consistency cycles of quasiparticle self-consistent calculations, small band gaps close to those measured experimentally $\left(E_{g, d}=13 \mathrm{meV}\right.$ and $E_{g, i}=$ $-33 \mathrm{meV}$ ) have been predicted [18]. In these calculations, the topologically trivial character of unstrained Bi crystal has been confirmed. Moreover, it has been shown that the Bi crystal can undergo semimetal to semiconductor and trivial to nontrivial phase transition under strains as small as $\sim 0.3 \%$.

In the present paper, we calculated the electronic energy band structures of 3D Bi with PBE, PBE+SOC, HSE, and HSE + SOC using the lattice constants, which are optimized within PBE + SOC. Calculated band structures are presented in Figs. 1(c) and 1(d). The values predicted for direct and indirect band gaps are listed in Table I. In the same table direct and indirect band gap calculated self-consistently using experimental lattice constants $[21,40]$ are also included for the sake of comparison. It is seen from the inset of Fig. 1 that $\mathrm{PBE}+\mathrm{SOC}$ and $\mathrm{HSE}+\mathrm{SOC}$ calculations carried out with optimized lattice constants predict $3 \mathrm{D} \mathrm{Bi}$ as semimetal and $E_{g, d}$ and $E_{g, i}$ in right order with experiment, but overestimate very small values of band gaps measured by $\mathrm{meV}$. The discrepancy between experimental values and those calculated by PBE + SOC increases even by $\sim 60 \%$ when calculations are carried out using experimental lattice constants. Even if the values of $E_{g, d}$ and $E_{g, i}$ calculated with experimental lattice constants and obtained after HSE+SOC correction reflect the right orders of experimental band gaps, these gaps are still overestimated. It appears that $\mathrm{PBE}+\mathrm{SOC}$ calculations, as well as HSE+SOC corrections cannot reproduce the small 
TABLE I. Direct $E_{g, d}$, and indirect $E_{g, i}$, band gaps of 3D Bi crystal calculated using PBE+SOC and HSE+SOC methods. The minus sign of $E_{g, i}$ denotes the inversion band edges. 3D hexagonal lattice constants are $a=b$ and $c$.

\begin{tabular}{lcccc}
\hline \hline & \multicolumn{2}{c}{ Optimized $a, c$} & Experimental $a, c$ \\
\cline { 2 - 5 } & $E_{g, d}(\mathrm{meV})$ & $E_{g, i}(\mathrm{meV})$ & $E_{g, d}(\mathrm{meV})$ & 100 \\
PBE+SOC & 60 & -119 & 38 & -185 \\
HSE+SOC & 87 & -40 & -133 \\
Experimental [18] & 13 & -33 & \\
\hline \hline
\end{tabular}

experimental band gaps of 3D $\mathrm{Bi}$ crystal in the range of $10 \mathrm{meV}$ (which is within the accuracy limits of DFT) and $-30 \mathrm{meV}$, even if one is able to be closer to them by adjusting HSE parameters. Accordingly, it may not be fruitful to discuss topologically trivial to nontrivial transitions of 3D Bi crystal having very small band gaps of a few meV using bare DFT results.

\section{SINGLE LAYER STRUCTURES OF BISMUTHENE}

Three suspended SL structures of bismuthene, namely buckled ( $b$-Bi), symmetric washboard ( $w$-Bi), and asymmetric washboard ( $a w-\mathrm{Bi})$ phases, are obtained by minimizing the total energies and atomic forces within PBE+SOC. Their optimized atomic configuration, lattice constants and relevant structure parameters are shown in Figs. 2(a)-4(a). When grown on suitable substrates, these SL phases of bismuthene are further stabilized.

\section{A. Optimized atomic structure and energetics}

The buckled bismuthene $b$-Bi has a 2D hexagonal lattice and honeycomblike structure with optimized lattice constants $a=b=4.38 \AA$. The lattice constants are compressed by $6 \%$ relative to those of $3 \mathrm{D} \mathrm{Bi}$ crystal. The $\mathrm{Bi}-\mathrm{Bi}$ bond length is

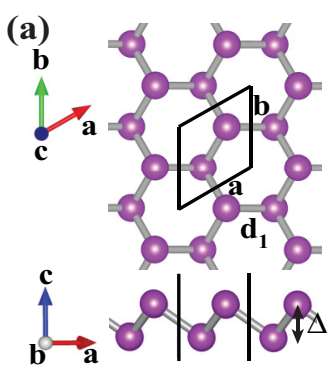

(b)

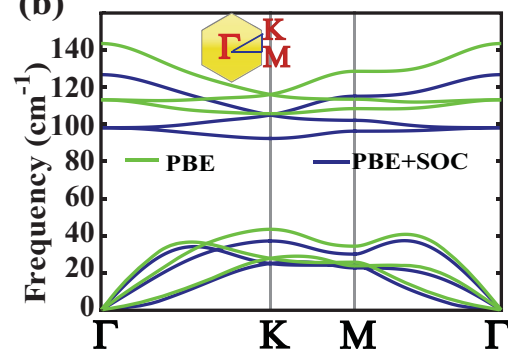

(c) $\mathbf{T}=300 \mathrm{~K}, 2 \mathrm{ps}$

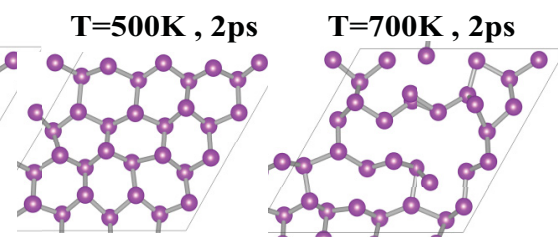

FIG. 2. Single layer of buckled honeycomb structure of $\mathrm{Bi}, b$-Bi: (a) top and side views of optimized atomic structure together with 2D hexagonal lattice constants $a=b$, buckling parameter $\Delta$. The 2D hexagonal unit cell is shaded. (b) Phonon dispersion curves are calculated with SOC (blue lines) and without SOC (green lines). (c) Snapshots of atomic structure calculated using $a b$ initio MD calculations carried out at $T=300,500$, and $700 \mathrm{~K}$. $d_{1}=3.07 \AA$. Three alternating atoms at the corners of the hexagon are raised, while the remaining three are lowered. Hence, the structure has two atomic planes; the spacing between these planes or buckling distance is $\Delta=1.74 \AA$, which is larger than the buckling of $3 \mathrm{D}$ crystal. This structure is stabilized by buckling, whereby planar $s p^{2}$ bonding is dehybridized to form $s p^{3}$-like hybridization to compensate for relatively weaker $\pi-\pi$ bonding that normally favors planar geometry in graphene. The cohesive energy $E_{C}=$ $1.95 \mathrm{eV} /$ atom indicates a significant binding. On the other hand, the formation energy $E_{f}=-0.132 \mathrm{eV} /$ atom is negative and hence is energetically $132 \mathrm{meV}$ less favorable relative to $3 \mathrm{D}$ crystal.

The symmetric washboard structure of bismuthene $w$-Bi, which is optimized by conjugate gradient method, has a single layer but consists of two atomic planes. The twodimensional rectangular lattice has four Bi atoms like black phosphorene. The in-plane and interplane $\mathrm{Bi}-\mathrm{Bi}$ bonds are found to be equal; $d_{1}=d_{2}=3.10 \AA$. The cohesive energy is $E_{C}=1.96 \mathrm{eV} /$ atom; it has $10 \mathrm{meV}$ stronger binding relative to $b$ - $\mathrm{Bi}$. Its negative formation energy at $T=0 \mathrm{~K}$ is $E_{f}=-0.12 \mathrm{eV} /$ atom. The asymmetric washboard bismuthene $a w$-Bi has four atomic planes and rectangular lattice. The in-plane and interplane $\mathrm{Bi}-\mathrm{Bi}$ bonds are $d_{1}=3.11 \AA$ and $d_{2}=3.10 \AA$, respectively. It has the cohesive energy $E_{C}=1.97 \mathrm{eV} /$ atom, which is highest among three SL bismuthene phases. The structural parameters, cohesive and formation energies, and other calculated values related to the mechanical properties of these SL-Bi phases are summarized in Table II. Notably, $b$ - $\mathrm{Bi}, w-\mathrm{Bi}$, and $a w$ - $\mathrm{Bi}$ have positive cohesive energies, but their formation energies are negative. Accordingly, these three SL structures correspond to local minima on the BO surface. Whether these SL structures are stable under small atomic displacements and whether these local minima are deep enough to assure structural stability at high temperature will be examined by extensive stability analysis in the next section.

\section{B. Stability analysis}

Even if the structure optimization using the conjugate gradient method favors stability of free-standing SL bismuthene phases with positive $E_{C}$, we perform further tests to assure they are stable in a local minimum and can sustain applications above the room temperature. First, to verify the stability of SL bismuthene phases against short and long wavelength, small transversal/longitudinal displacements in different directions at $T=0 \mathrm{~K}$, we calculate frequencies of vibrational modes of $b$ - $\mathrm{Bi}, w$ - $\mathrm{Bi}$, and $a w$ - $\mathrm{Bi}$ using a fine graded k-point sampling. 
TABLE II. Calculated values of SL bismuthene phases: lattice constants $a$ and $b$; bond lengths $d_{1}$ and $d_{2}$; cohesive $E_{C}$ and formation $E_{f}$ energies; components of in-plane stiffness $C_{x}, C_{y}$; Poisson's ratio $v_{x y}, v_{y x}$.

\begin{tabular}{|c|c|c|c|c|c|c|c|c|c|}
\hline Structure & $\mathrm{a}(\AA)$ & $\mathrm{b}(\AA)$ & $d_{1}(\AA)$ & $d_{2}(\AA)$ & $\Delta(\AA)$ & $\mathrm{C}(\mathrm{N} / \mathrm{m})$ & $\begin{array}{c}E_{C}(\mathrm{eV} / \\
\text { atom })\end{array}$ & $\begin{array}{c}E_{f}(\mathrm{meV} / \\
\quad \text { atom })\end{array}$ & $v$ \\
\hline$b-\mathrm{Bi}$ & $\begin{array}{c}4.38 \\
4.39[47]\end{array}$ & $\begin{array}{c}4.38 \\
4.39[47]\end{array}$ & 3.07 & & $\begin{array}{c}1.74 \\
1.68[47]\end{array}$ & $\mathrm{C}_{x}=\mathrm{C}_{y}=23.9$ & 1.954 & -132 & $v_{x y}=0.327 ; v_{y x}=0.327$ \\
\hline$w$-Bi & 5.15 & 4.51 & 3.10 & 3.10 & & $\mathrm{C}_{x}=8.04, \mathrm{C}_{y}=22.6$ & 1.966 & -120 & $v_{x y}=0.333 ; v_{y x}=0.933$ \\
\hline$a w-\mathrm{Bi}$ & $\begin{array}{c}4.94 \\
(4.87[48]) \\
(4.78[49]) \\
(4.75[50]) \\
(4.9[21])\end{array}$ & $\begin{array}{c}4.55 \\
(4.44[48]) \\
(4.40[49]) \\
(4.54[50]) \\
(4.5[21])\end{array}$ & $\begin{array}{c}3.11 \\
(3.1[21])\end{array}$ & 3.10 & $\begin{array}{c}0.5 \\
(0.5[49]) \\
(0.32[50])\end{array}$ & $\mathrm{C}_{x}=10.03, \mathrm{C}_{y}=25.5$ & 1.971 & -115 & $v_{x y}=0.261 ; v_{y x}=0.648$ \\
\hline
\end{tabular}

The stability of a bismuthene phase at low temperature will be assured if only vibrational frequencies calculated for all $\mathbf{k}$ points are positive. Otherwise, imaginary frequencies would imply structural instability for corresponding modes. Dispersion of vibration frequencies of free-standing SL $b-\mathrm{Bi}$, $w$ - $\mathrm{Bi}$, and $a w$ - $\mathrm{Bi}$ structures calculated using $\mathrm{PBE}+\mathrm{SOC}$ are presented along the major symmetry axis in Figs. 2, 3, and 4(b). The vibrational frequencies calculated using only PBE are also presented to reveal the effect of SOC on the frequencies.

In Fig. 2(b) the vibrational frequencies of $b$-Bi are all positive. In particular, the frequencies of acoustical, flexural ZA modes are positive as $\mathbf{k} \rightarrow 0$ ensures stability even for long wavelength lattice vibrations. The optical branches are separated from acoustical branches. The effect of SOC on phonon bands appears as down shifts [51] of optical frequencies by $\Delta \Omega=0.42 \mathrm{THz}$. Moreover, acoustical modes are slightly softened upon inclusion of SOC. While present calculations predict optical phonon frequencies at the $\Gamma$ point of the BZ as $126.73 \mathrm{~cm}^{-1}$ and (doubly degenerate) $98.18 \mathrm{~cm}^{-1}$, these frequencies were calculated as $109.68 \mathrm{~cm}^{-1}$ and (two split frequencies) $95.17 \mathrm{~cm}^{-1}, 98.39 \mathrm{~cm}^{-1}$ using density functional perturbation theory [51]. Notably, these LO-TO split frequencies are predicted as degenerate by present DFT calculations.

The vibrational frequencies of $w$-Bi in Fig. 3(b) have imaginary frequencies near the $\Gamma$ point of the ZA branch, which is an artifact of numerical accuracy occurring due to limited size of supercell used in calculations. However, (a)

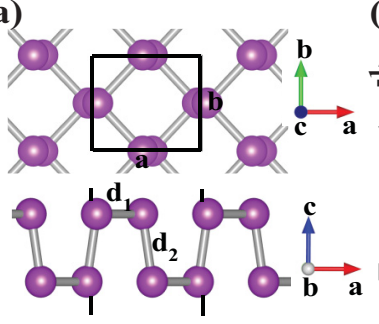

(b)

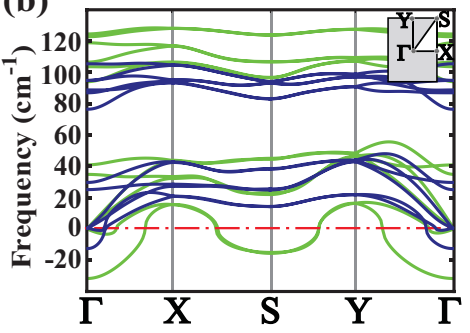

FIG. 3. Single layer of symmetric washboard structure of Bi, $w$-Bi: (a) Top and side views of optimized atomic structure together with lattice constants. The 2D rectangular unit cell is shaded. (b) Phonon dispersion curves are calculated with SOC (blue lines) and without SOC (green lines). imaginary frequencies of an optical branch in a significantly large region of the $\mathrm{BZ}$ and its mixing with acoustical branches are serious and imply an instability. Displacements of atoms from their equilibrium positions are then deprived of the restoring force. This indicates that $w$ - $\mathrm{Bi}$ structure is unstable and will spontaneously transform to a different structure. In fact, the atomic structure and lattice constants are optimized concomitantly, and $w$-Bi transforms to $a w$-Bi spontaneously. A similar situation also occurred for the sw-antimonene [5]. Nevertheless, even if $w$-Bi appears to be unstable, it can be stable when its size is reduced or it is placed on a substrate. For this reason, the mechanical and electronic properties of $w$-Bi will be discussed in forthcoming sections.

All calculated frequencies of the $a w$-Bi structure are positive and hence indicate the stability against small displacement of atoms in all directions. In contrast to $b$-Bi structure, three optical branches occur just above the acoustical branches and overlap with them along specific directions. The effect of SOC on the vibrational frequencies is deduced by significant downshifts of optical frequencies, namely as phonon softening.

(a)
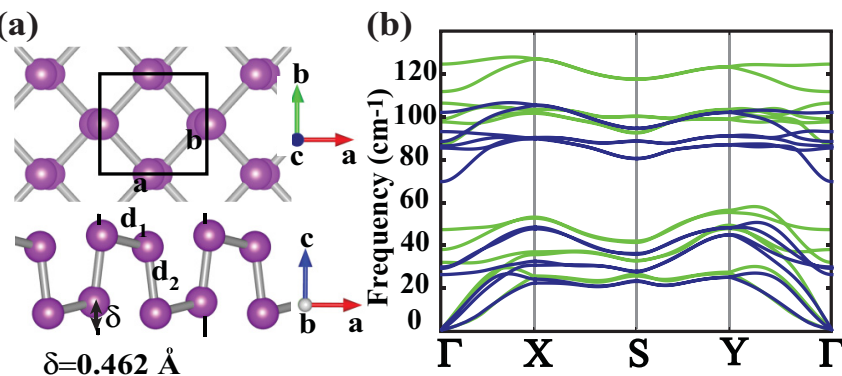

(c) $\mathrm{T}=300 \mathrm{~K}, 2 \mathrm{ps}$
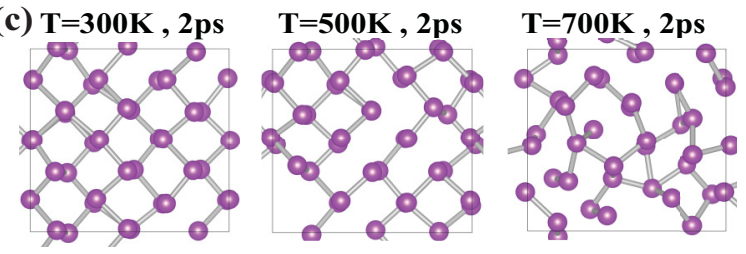

FIG. 4. Single layer of asymmetric washboard structure of $\mathrm{Bi}$, $a w$-Bi: (a) Top and side views of optimized atomic structure together with lattice constants. The 2D rectangular unit cell is shaded. (b) Phonon dispersion curves are calculated with SOC (blue lines) and without SOC (green lines). (c) Snapshots of atomic structure calculated using $a b$ initio MD calculations carried out at $T=300$, 500 , and $700 \mathrm{~K}$. 
One concludes from these results that nitrogene has only stable single-layer buckled honeycomb structure [1], phosphorene [3] and arsenene [4] have buckled honeycomb and symmetric washboard structures, and antimonene [5] and bismuthene have buckled honeycomb and asymmetric washboard structures.

Even if all acoustical including ZA branches with positive frequencies ensure stability, the optimized structure by itself may correspond to a local shallow minimum, and hence it may turn to be unstable by thermal excitations at elevated temperatures. We clarified this critical situation by performing $a b$ initio MD calculations starting from $300 \mathrm{~K}$ up to $1000 \mathrm{~K}$. The stability of these structures are maintained at high temperatures, but the instability due to thermal excitations occurred at $T=700 \mathrm{~K}$ as depicted from the MD snapshots of atomic structure in Figs. 2 and 4(c).

\section{Mechanical properties}

We investigated mechanical properties by calculating [52] in-plane stiffness, $C=(1 / A) \partial E_{T}^{2} / \partial \epsilon^{2}$, and Poisson's ratio $v_{x y}=-\epsilon_{y} / \epsilon_{x}$ for the SL phases of bismuthene. In-plane stiffness of $b$-Bi homogeneous and $C_{x}=C_{y}=23.9 \mathrm{~N} / \mathrm{m}$. Both $w$ - $\mathrm{Bi}$ and $a w$ - $\mathrm{Bi}$ display directional in-plane stiffness values. For $w$-Bi $C_{x}=8.0 \mathrm{~N} / \mathrm{m}$ and $C_{y}=22.6 \mathrm{~N} / \mathrm{m}$. The calculated in-plane stiffness values of $a w$-Bi are $C_{x}=10.0 \mathrm{~N} / \mathrm{m}$ and $C_{y}=25.5 \mathrm{~N} / \mathrm{m}$, which are higher than those of $w$-Bi. Here the calculated values of in-plane stiffness of SL bismuthene are found to be small when compared with those of graphene $(330 \mathrm{~N} / \mathrm{m}), \mathrm{h}-\mathrm{BN}(240 \mathrm{~N} / \mathrm{m})$, silicene $(65 \mathrm{~N} / \mathrm{m})$, and $\mathrm{MoS}_{2}$ $(138 \mathrm{~N} / \mathrm{m})$, all having single-layer honeycomb structure. Poisson's ratio of $b$ - $\mathrm{Bi}$ is uniform and has a usual value. However, the calculated values of Poisson's ratio of $w$-Bi and $a w-\mathrm{Bi}$ are directional. Along the $x$ direction $v_{x y}$ has usual values, but the calculated values of $v_{y x}$ are rather high as presented in Table II.

\section{ELECTRONIC STRUCTURE}

Recent studies have demonstrated that SL structures of other group V elements, nitrogene [1], phosphorene [3], arsenene [4], and antimonene [5] are semiconductors. This property has made SL structures of group $\mathrm{V}$ elements an active field of study. While 3D Bi crystal is a semimetal and is a $3 \mathrm{D}$ topological insulator, the electronic properties of SL bismuthene are of particular interest. Earlier studies have focused on the $2 \mathrm{D}$ topological insulator aspects of very thin $\mathrm{Bi}$ films. Having revealed the optimized atomic structures of SL bismuthene phases and their stability above room temperature, here we investigate their electronic energy band structure. Since the spin-orbit coupling in 3D Bi crystal is known to be crucial, here we investigate the effects of SOC and HSE corrections on the electronic energy structures of $b-\mathrm{Bi}, w-\mathrm{Bi}$, and $a w-\mathrm{Bi}$ which were calculated within PBE. To this end, we present the electronic band structures of bismuthene phases calculated by PBE, PBE+SOC, HSE and HSE+SOC in Fig. 5. In Table III we also list the band gaps calculated by PBE and HSE with and without SOC. All the calculations have been carried out using lattice constants optimized by PBE+SOC.

Calculations using PBE predict a direct band gap of $E_{g}=$ $0.55 \mathrm{eV}$ for the optimized structure of SL $b$-Bi at the center
TABLE III. Calculated direct (d) and indirect (i) band gaps of free-standing SL bismuthene phases. HSE corrections are performed with $\alpha=0.25$.

\begin{tabular}{lcc}
\hline \hline Structure & $\begin{array}{c}E_{g-P B E} \\
E_{g-P B E+S O C}(\mathrm{eV})\end{array}$ & $\begin{array}{c}E_{g-H S E} \\
E_{g-H S E+S O C}(\mathrm{eV})\end{array}$ \\
\hline$b-\mathrm{Bi}$ & $0.547(\mathrm{~d})$ & $0.803(\mathrm{~d})$ \\
& $0.505(\mathrm{i})$ & $0.317(\mathrm{~d})$ \\
$w-\mathrm{Bi}$ & $0.160(\mathrm{~d})$ & $0.144(\mathrm{~d})$ \\
& $0.164(\mathrm{i})$ & $0.027(\mathrm{~d})$ \\
$a w-\mathrm{Bi}$ & $0.306(\mathrm{~d})$ & $0.229(\mathrm{~d})$ \\
& $0.280(\mathrm{i})$ & $0.386(\mathrm{i})$ \\
\hline \hline
\end{tabular}

of the hexagonal Brillouin zone. The band gap is larger than $3 \mathrm{eV}$ at the $K$ point. The conduction band has high effective mass at $\mathbf{k}=0$. This situation, however, changes when SOC is included. The degenerate bands at the top of the valence band at the $\Gamma$ point have a Rashba-type splitting and reduce the band gap to $0.51 \mathrm{eV}$ by changing its character to indirect. Moreover, the effective mass of the conduction band electrons reduces and the degenerate bands split. The HSE correction applied to PBE states increases the direct band gap to $0.80 \mathrm{eV}$. However, when SOC is included, this fundamental gap decreases to $0.32 \mathrm{eV}$, and the doubly degenerate bands at the $\Gamma$ point split
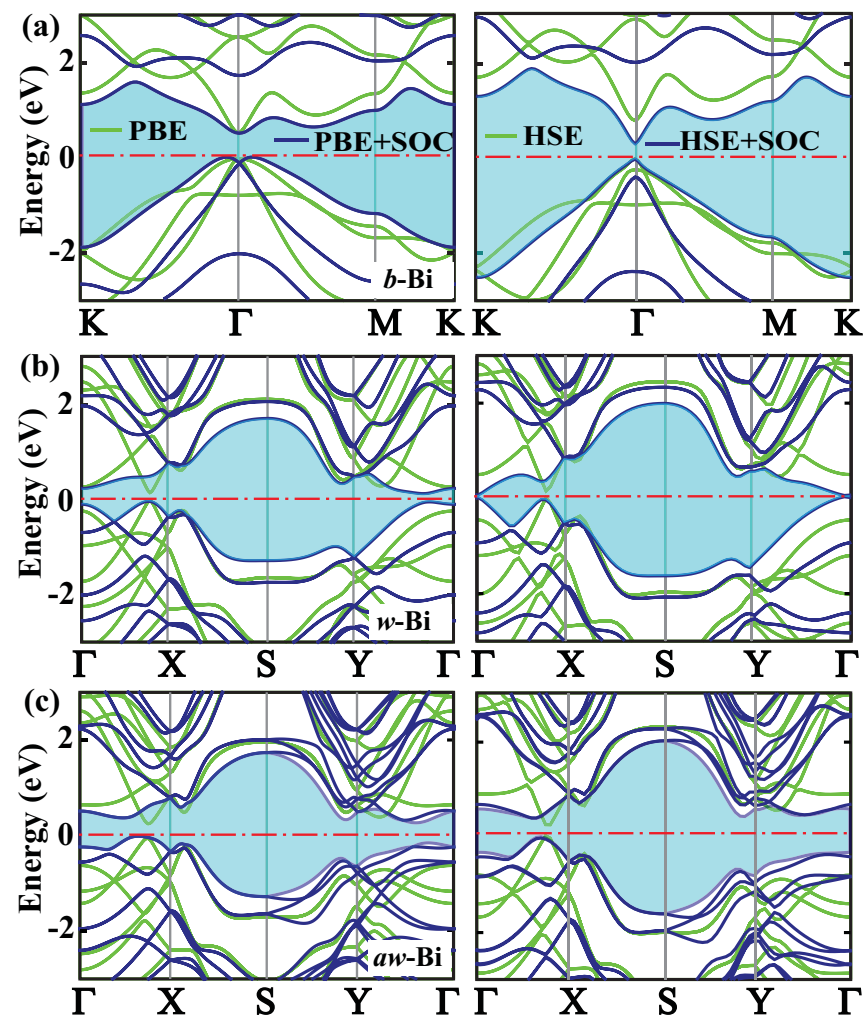

FIG. 5. Electronic energy band structures of free-standing SL bismuthene phases. (a) SL $b$-Bi. (b) SL $w$-Bi. (c) SL $a w$-Bi. Left panels are calculated by PBE (green lines) and $\mathrm{PBE}+\mathrm{SOC}$ (blue lines); right panels are calculated by HSE (green lines) and HSE+SOC (blue lines). The zero of energy is set to the top of the valence bands. The gap between conduction and valence bands is shaded. 
by $\sim 0.3 \mathrm{eV}$. Briefly, in contrast to the 3D Bi crystal SL, $b$-Bi is a narrow band gap, nonmagnetic semiconductor. It appears that by going from 3D $\mathrm{Bi}$ crystal to monolayer $b$-Bi lattice constants are compressed; the buckling parameter is slightly increased but a narrow band gap is opened. As will be clarified, the absence of the interlayer interaction causes a band gap to open in SL $b$-Bi. Earlier, calculations of the band structure of SL $b$-Bi using DFT with the full potential linearized augmented plane wave method predicted that $\mathrm{SL} b$-Bi is a semiconductor, even if the band gap is decreased with the inclusion of SOC [22]. This trend agrees with present findings. Band gap values calculated by Freitas et al. [30] using PBE with and without SOC agree with the present values.

The minimum band gaps of $w$ - and $a w$-Bi calculated with PBE (without SOC) occur along the $\Gamma-X$ direction. The calculated values of these direct band gaps are $E_{g}=0.16 \mathrm{eV}$ and $E_{g}=0.31 \mathrm{eV}$ for $w$ - and $a w-\mathrm{Bi}$, respectively. Upon inclusion of SOC, these band gaps of $w$ - and $a w$-Bi become indirect and attain the similar values obtained without SOC; namely $E_{g}=0.16 \mathrm{eV}$ and $E_{g}=0.28 \mathrm{eV}$, respectively. When corrected with HSE method (without SOC), the direct band gap of $w$ - $\mathrm{Bi}$ is decreased to $E_{g}=0.14 \mathrm{eV}$. However, this band gap is almost closed at the $\Gamma$ point upon inclusion of SOC to HSE correction. The PBE band gap of $a w-\mathrm{Bi}$ decreases to $E_{g}=0.23 \mathrm{eV}$ when corrected by HSE without SOC. Upon inclusion of SOC to HSE correction, the band gap of $a w$-Bi becomes indirect and attains the value of $0.39 \mathrm{eV}$. Like $b-\mathrm{Bi}, a w-\mathrm{Bi}$, which is the most stable structure among $\mathrm{SL}$ bismuthene, is a narrow band gap material. While the above HSE + SOC band gap values are calculated with $\alpha=0.25$, depending on the value of the mixing parameter $0<\alpha<1.0$, the band gap value ranges between 0.52 and $0.99 \mathrm{eV}$ in $b-\mathrm{Bi}$, between 0.16 and $2.28 \mathrm{eV}$ in $w-\mathrm{Bi}$, and between 0.30 and $2.21 \mathrm{eV}$ in $a w-\mathrm{Bi}$.

\section{BILAYERS OF BISMUTHENE PHASES}

The thin films of SL bismuthene phases are of interest for possible technological applications. Here we investigated bilayers (BL) of SL $b$-Bi and $a w$-Bi phases. The stacking sequence and corresponding atomic structure is determined for both SL phases by the minimization of the total energy, atomic forces, and pressure among different stacking sequences.

For $\mathrm{BL} b$-Bi the AA stacking has been found to be most favorable energetically. The binding energy between single layers in $\mathrm{BL}$ is calculated to be $136 \mathrm{meV}$ [without van der Waals (vdW) correction]. The distance between similar $\mathrm{Bi}$ atoms in different layers is $4.80 \AA$ and the minimum spacing between $\mathrm{Bi}$ atomic planes is $z=3.09 \AA$, which are $0.95 \AA$ and $0.65 \AA$ larger, respectively, than those in 3D Bi crystal. The hexagonal lattice constants $a=b=4.42 \AA$ are larger than those of SL $b$-Bi but still smaller than those of 3D Bi crystal ( $a=b=4.64 \AA)$. We note that the chemical interaction dominates the binding between two layers in $\mathrm{BL}$ $b$ - $\mathrm{Bi}$; inclusion of $\mathrm{vdW}$ correction [53] to the total energy underestimates the interlayer distance and overestimates the cohesive energy.

The energy band structures of $\mathrm{BL}, b$-Bi are calculated by using PBE, PBE+SOC, HSE, and HSE+SOC in order to reveal the effects of SOC and band correction on PBE
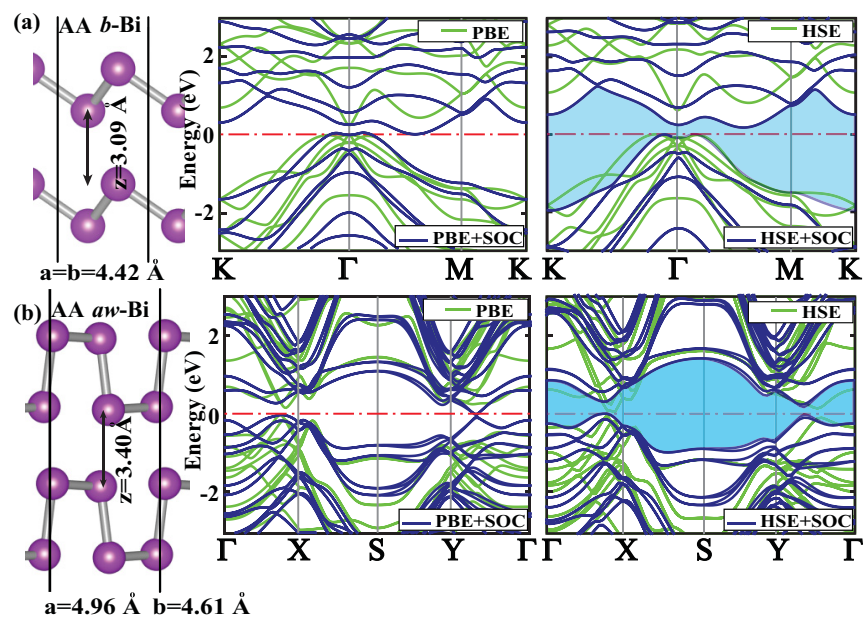

FIG. 6. Atomic and electronic energy band structure of bilayers (BL) of bismuthene phases (i.e., two SL bismuthene) with minimum energy stacking sequence. (a) $\mathrm{BL}$ of $b$-Bi with AA stacking where similar $\mathrm{Bi}$ atoms in different layers are situated on top of each other. (b) BL of $a w$-Bi with AA stacking. Left: Energy band structure calculated by using PBE (green lines) and by PBE+SOC (blue lines). Right: Bands are calculated using HSE (green lines) and by HSE+SOC (blue lines).

results. The calculated energy band structures are presented in Fig. 6(a). PBE calculations predict that $\mathrm{BL} b$-Bi is a direct band gap semiconductor with a band gap $E_{g}=0.23 \mathrm{eV}$, which changes to metal after SOC is included. After HSE correction to PBE results, the indirect band gap becomes $E_{g}=0.61 \mathrm{eV}$, which is reduced to $0.24 \mathrm{eV}$ in $\mathrm{HSE}+\mathrm{SOC}$ calculations. Hence both $\mathrm{PBE}+\mathrm{SOC}$ and $\mathrm{HSE}+\mathrm{SOC}$ band gaps of $\mathrm{BL}-\mathrm{Bi}$ are finite but smaller than those of SL $b$-Bi. It appears that the transition from semiconductor to semimetal can occur in multilayers of $b$-Bi. The full potential linearized augmented plane wave method within DFT predicts semimetallic and metallic states with and without SOC, respectively, and hence disagrees with present $\mathrm{PBE}+\mathrm{SOC}$ and $\mathrm{HSE}+\mathrm{SOC}$ results.

The band structure of BL $a w$-Bi presented in Fig. 6(b) exhibits features similar to those of $\mathrm{BL} b$-Bi. The AA stacking is also preferred by $\mathrm{BL} a w-\mathrm{Bi}$ with minimum spacing of $3.40 \AA$. The binding energy between single layers in $\mathrm{BL}$ aw-Bi is calculated to be $399 \mathrm{meV}$. The PBE band is calculated to be indirect and $E_{g}=0.18 \mathrm{eV}$, which is smaller than the gap in SL $a w$-Bi. Upon inclusion of SOC, the gap is closed and $\mathrm{BL} a w$-Bi becomes a metal. However, the band gap is opened when PBE bands are corrected by HSE, where the gap attains the value of $0.09 \mathrm{eV}$. This gap increases to $0.18 \mathrm{eV}$ when SOC is included. Like $\mathrm{BL} b$-Bi, BL $a w$-Bi has also a small band gap of $E_{g}=0.18 \mathrm{eV}$ predicted by $\mathrm{HSE}+\mathrm{SOC}$ calculations, which is smaller than the corresponding gap of $\mathrm{SL} a w-\mathrm{Bi}$. By increasing the number of layers the band gap is expected to reduce, and the transition from semiconductor to metal may occur in multilayer structures with inverted edges of conduction and valence band edges.

\section{CONCLUSIONS}

In conclusion, using first-principles phonon and finite temperature molecular dynamic calculations we demonstrated 
that single-layer buckled and asymmetric washboard phases of bismuth are stable against long wavelength vibrations and thermal excitations at high temperature. Hence, these 2D phases correspond to local minima in the Born-Oppenheimer surface and are nonmagnetic, narrow band semiconductors. While 3D hexagonal $\mathrm{Bi}$ crystal with a small direct band gap and inverted indirect band gap is a semimetal, its single-layer buckled honeycomb structure is a narrow band gap semiconductor. A similar transition from semimetal to semiconductor is also shared by the single-layer asymmetric washboard structure. On the other hand, by going from single-layer to bilayer bismuthene fundamental band gap is decreased and probably closed in multilayer structures. This result indicates the importance of interlayer interaction in multilayers and single layers from topologically nontrivial to trivial and semimetal-semiconductor transitions in $\mathrm{Bi}$. The electronic band gap can be tuned by layer thickness. Like 3D Bi crystal, single-layer as well as multilayer bismuthene phases display interesting features apart from topological characters and require further theoretical and experimental studies. In particular, the properties of these layered structures on selected substrates and their functionalization through adsorption with selected adatoms are of current interest.

\section{ACKNOWLEDGMENTS}

The authors of this paper thank Dr. Mehmet Topsakal for his illuminating suggestions and fruitful discussions. The computational resources are provided by TUBITAK ULAKBIM, High Performance and Grid Computing Center (TR-Grid e-Infrastructure). E.A. and S.C. acknowledge financial support from the Academy of Sciences of Turkey (TÜBA).
[1] V. O. Özçelik, O. U. Aktürk, E. Durgun, and S. Ciraci, Phys. Rev. B 92, 125420 (2015).

[2] L. Li, Y. Yu, G. J. Ye, Q. Ge, X. Ou, H. Wu, D. Feng, X. H. Chen, and Y. Zhang, Nat. Nanotechnol. 9, 372 (2014).

[3] Z. Zhu and D. Tomanek, Phys. Rev. Lett. 112, 176802 (2014).

[4] C. Kamal and M. Ezawa, Phys. Rev. B 91, 085423 (2015).

[5] O. U. Aktürk, V. O. Özçelik, S. Ciraci, Phys. Rev. B 91, 235446 (2015).

[6] Z. Zhang, Z. Yan, Y. Li, Z. Chen, and H. Zeng, Angew. Chem. Int. Ed. 54, 3112 (2015).

[7] K. S. Novoselov, A. K. Geim, S. V. Morozov, D. Jiang, M. I. Katsnelson, I. V. Grigorieva, S. V. Dubonos, and A. A. Firsov, Nature (London) 438, 197 (2005).

[8] E. Durgun, S. Tongay, and S. Ciraci, Phys. Rev. B 72, 075420 (2005).

[9] S. Cahangirov, M. Topsakal, E. Aktürk, H. Sahin, and S. Ciraci, Phys. Rev. Lett. 102, 236804 (2009).

[10] C. R. Ast and H. Höchst, Phys. Rev. B 67, 113102 (2003).

[11] Y. M. Koroteev, G. Bihlmayer, J. E. Gayone, E. V. Chulkov, S. Blügel, P. M. Echenique, and Ph. Hofmann, Phys. Rev. Lett. 93, 046403 (2004).

[12] E. J. Tichovolsky and J. G. Mavroides, Solid State Commun. 7, 927 (1969)

[13] Y. Ohtsubo, L. Perfetti, M. O. Goerbig, P. L. Fevre, F. Betran, and A. Taleb-Ibrahimi, New J. Phys. 15, 033041 (2013).

[14] C. L. Kane and E. J. Mele, Phys. Rev. Lett. 95, 226801 (2005).

[15] M. Z. Hasan and C. L. Kane, Rev. Mod. Phys. 82, 3045 (2010).

[16] J. E. Moore, Nature (London) 464, 194 (2010).

[17] Topoloical Insulators, edited by F. Ortmann, S. Roche and S. O. Valenzuela (Wiley-VCH, Weinheim, Germany, 2015).

[18] I. Aguilera, C. Friedrich, and S. Blügel, Phys. Rev. B 91, 125129 (2015).

[19] H. Zhang, C-X. Liu, X.-L. Qi, X. Dai, Z. Fang, and S-C. Zhang, Nat. Phys. 5, 438 (2009).

[20] D. Hsieh, D. Qian, L. Wray, Y. Xia, Y. S. Hor, R. J. Cava, and M. Z. Hasan, Nature (London) 452, 970 (2008).

[21] T. Nagao, J. T. Sadowski, M. Saito, S. Yaginuma, Y. Fujikawa, T. Kogure, T. Ohno, Y. Hasegawa, S. Hasegawa, and T. Sakurai, Phys. Rev. Lett. 93, 105501 (2004).
[22] Y. M. Koroteev, G. Bihlmayer, E. V. Chulkov, and S. Blügel, Phys. Rev. B 77, 045428 (2008).

[23] S. Yaginuma, K. Nagaoka, T. Nagao, G. Bihlmayer, Y. M Koroteev, E. V. Chulkov, and T. Nakayama, J. Phys. Soc. Jpn. 77, 014701 (2008).

[24] M. Wada, S. Murakami, F. Freimuth, and G. Bihlmayer, Phys. Rev. B 83, 121310 (2011).

[25] Z. Liu, C.-X. Liu, Y.-S. Wu, W.-H. Duan, F. Liu, and J. Wu, Phys. Rev. Lett. 107, 136805 (2011).

[26] F. Yang, L. Miao, Z. F. Wang, M-.Y. Yao, F. Zhu, Y. R. Song, M.-X. Wang, J.-P. Xu, A. V. Fedorov, Z. Sun, G. B. Zhang, C. Liu, F. Liu, D. Qian, C. L. Gao, and J.-F. Jia, Phys. Rev. Lett. 109, 016801 (2012).

[27] L. Chen, Z. F. Wang, and F. Liu, Phys. Rev. B 87, 235420 (2013).

[28] L. Cheng, H. Liu, X. Tan, J. Zhang, J. Wei, H. Lv, J. Shi, and X. Tang, J. Phys. Chem. C 118, 904 (2014).

[29] H. Iwasaki and T. Kikegawa, Acta Crystallogr. B. 53, 353 (1997).

[30] R. R. Freitas, R. Rivelino, F. de B. Mota, and C. M. C. de Castilho, J. Phys. Chem. C, 119, 23599 (2015).

[31] J. Heyd, G. E. Scuseria, and M. Ernzerhof, J. Chem. Phys. 118, 8207 (2003).

[32] W. Kohn and L. J. Sham, Phys. Rev. 140, A1133 (1965).

[33] P. E. Blöchl, Phys. Rev. B 50, 17953 (1994).

[34] J. P. Perdew, K. Burke, and M. Ernzerhof, Phys. Rev. Lett. 77, 3865 (1996).

[35] G. Kresse and J. Furthmüller, Phys. Rev. B 54, 11169 (1996).

[36] H. J. Monkhorst and J. D. Pack, Phys. Rev. B 13, 5188 (1976).

[37] A. Togo and I. Tanaka, Scr. Mater. 108, 1 (2015).

[38] S. Nosé, J. Chem. Phys. 81, 511 (1984).

[39] X. Gonze, J.-P. Michenaud, and J.-P. Vigneron, Phys. Rev. B 41, 11827 (1990).

[40] H. Mönig, J. Sun, Yu M. Koroteev, G. Bihlmayer, J. Wells, E. V. Chulkov, K. Pohl, and Ph. Hofmann, Phys. Rev. B 72, 085410 (2005).

[41] C. Kittel, Introduction to Solid State Physics, 8th ed. (John Wiley and Sons, New York, 1996).

[42] K. Ishioka, M. Kitajima, O. V. Misochko, and T. Nagao, Phys. Rev. B 91, 125431 (2015).

[43] L. E. Díaz-Sánchez, A. H. Romero, and X. Gonze, Phys. Rev. B 76, 104302 (2007). 
[44] G. Q. Huang and J. Yang, J. Phys.: Condens. Matter, 25, 175004 (2013).

[45] P. Hofmann, Prog. Surf. Sci. 81, 191 (2006).

[46] R. N. Brown, J. G. Movroides, and B. Lax, Phys. Rev. 129, 2055 (1963); G. E. Smith, G. A. Baraff, and J. M. Rowell, ibid. 135, A1118 (1964); R. T. Isaacson and G. A. Williams, ibid. 185, 682 (1969); M. Maltz and M. S. Dresselhaus, Phys. Rev. B 2, 2877 (1970); M. P. Vecchi and M. S. Dresselhaus, ibid. 10, 771 (1974).

[47] T. Hirahara, N. Fukui, T. Shirasawa, M. Yamada, M. Aitani, H. Miyazaki, M. Matsunami, S. Kimura, T. Takahashi, S. Hasegawa, and K. Kobayashi, Phys. Rev. Lett. 109, 227401 (2012).
[48] P. J. Kowalczyk, O. Mahapatra, D. Belic, S. A. Brown, G. Bian, and T.-C. Chiang, Phys. Rev. B 91, 045434 (2015).

[49] Y. Lu, W. Xu, M. Zheng, G. Yao, L. Shen, M. Yang, Z. Luo, F. Pan, K. Wu, T. Das, J. Jiang, J. Martin, Y. P. Feng, H. Lin, and X-Sen Wang, Nano Lett. 15, 80 (2015).

[50] J.-T. Sun, H. Huang, S. L. Wong, H.-J. Gao, Y. P. Feng, and A. T. S. Wee, Phys. Rev. Lett. 109, 246804 (2012).

[51] V. Chis, G. Benedek, P. M. Echenique, and E. V. Chulkov, Phys. Rev. B. 87, 075412 (2013).

[52] M. Topsakal, S. Cahangirov, and S. Ciraci, Appl. Phys. Lett. 96, 091912 (2011).

[53] S. Grimme, J. Comput. Chem. 27, 1787 (2006). 